\title{
Importance of Threshold Corrections in Quark-Lepton Complementarity
}

\author{
Sin Kyu Kang \\ School of Physics, Seoul National University, Seoul 151-741, Korea \\ C. S. Kim and Jake Lee \\ Department of Physics, Yonsei University, Seoul 120-749, Korea
}

(Dated: October 12, 2018)

\begin{abstract}
The recent experimental measurements of the solar neutrino mixing angle $\theta_{\text {sol }}$ and the Cabibbo mixing angle $\theta_{C}$ reveal a surprising relation, $\theta_{\text {sol }}+\theta_{C} \simeq \frac{\pi}{4}$. We note that the lepton mixing matrix derived from quark-lepton unification can lead to a shift of the complementarity relation at low energy. While the renormalization group effects generally lead to additive contribution on top of the shift, in this letter, we show that the threshold corrections which may exist in some intermediate scale new physics such as supersymmetric standard model can diminish it, so we can achieve the complementarity relation at a low energy. Finally, we discuss a possibility to achieve the complementarity relation at a high energy by taking particular form of non-symmetric form of down Yukawa matrix.
\end{abstract}

PACS numbers: 14.60.Pq,12.15.Ff,12.10.Dm,11.10.Gh 
Recently, it has been noted that the solar neutrino mixing angle $\theta_{\text {sol }}$ required for a solution of the solar neutrino problem and the Cabibbo angle $\theta_{C}$ reveal a surprising relation

$$
\theta_{\text {sol }}+\theta_{C} \simeq \frac{\pi}{4}
$$

which is satisfied by the experimental results

$\theta_{\text {sol }}+\theta_{C}=45.4^{\circ} \pm 1.7^{\circ}$ to within a few percent accuracy [1, 2, 3]. This quark-lepton complementarity (QLC) relation (1) has been interpreted as an evidence for certain quarklepton symmetry or quark-lepton unification as shown in Refs. [4, 5, 6]. Yet, it can be a coincidence in the sense that reproducing the exact QLC relation (1) at low energy scale in the framework of grand unification depends on the renormalization effects whose size can vary with the choice of parameter space. But, we believe that such a coincidence does not necessarily mean that attempts to catch a deep meaning behind the QLC relation were in vain. Anyway, establishing the origin of the QLC relation may be a challenge that underlying theory of flavor should address.

To effectively describe the deviation from maximal mixing of solar neutrino, small mixing element $U_{e 3}$ and possible deviation from maximal mixing of atmospheric neutrino, a parametrization of the PMNS mixing matrix in terms of a small parameter whose magnitude can be interestingly around $\sin \theta_{C}$ has been proposed as follows [7, 8, 9, 10]:

$$
U_{\mathrm{PMNS}}=U^{\dagger}(\lambda) U_{\text {bimax }}
$$

Here $U(\lambda)$ is a mixing matrix parameterized in terms of a small parameter $\lambda$ and $U_{\text {bimax }}$ corresponds to the bi-maximal mixing matrix [11]. Among possible generic structures of the matrix $U(\lambda)$ which are compatible with experimental results on the neutrino oscillations, the "CKM-like" form of $U(\lambda)$ has rather profound implication in view of the connection between quarks and leptons.

In this letter, we first of all show that the lepton mixing matrix given in the form of Eq. (2) with the "CKM-like" $U(\lambda) \sim U_{\mathrm{CKM}}$ can be indeed realized in the framework of grand unification with symmetric Yukawa matrices when we incorporate seesaw mechanism, and examine whether or not $U_{\text {PMNS }}$ reflecting quark-lepton unification given by (2) can predict the QLC relation (1) exactly. We see that the solar mixing angle derived from $U_{\text {PMNS }}$ leads to correction to the QLC relation which can be more than $1 \sigma$ deviation from the QLC relation as similarly shown in [5]. Notice that while the QLC relation holds at a low energy, the corresponding relation derived from the mixing matrix given in the form of Eq. (2) is in fact realized at a high scale such as seesaw scale or unification scale. Thus, it is necessary to take into account the renormalization effects on the lepton mixing matrix when one compares the prediction at a high energy scale with the QLC relation observed at low energy scale. One can then expect that the deviation from the QLC relation is explained by renormalization effects. In the SM, the renormalization effect through the renormalization group ( $R G$ ) running down to the weak scale is negligible because of small charged lepton Yukawa couplings. But, in some models such as the minimal supersymmetric standard model (MSSM), the renormalization effect on the leptonic mixing angle $\theta_{12}$ depends on the type of light neutrino mass spectrum as well as model parameters [12, 13, 14]. It is also known that in MSSM with large $\tan \beta$ and the quasi-degenerate neutrino mass spectrum the RG effects are generally large and can enhance the mixing angle $\theta_{12}$ at low energy [12, 13, 14]. Such an enhancement of $\theta_{12}$ is not suitable for achieving the QLC relation (1) at low energy because we need to diminish the deviation so as to get the exact QLC relation (1) at a low energy. 
In this letter, we show that the sizeable threshold corrections which may exist in the MSSM [15, 16, 17] can diminish the deviation from the QLC relation while keeping the mixing angle $\theta_{23}$ almost maximal and $\theta_{13}$ small, so that the QLC relation at low energy can be achieved in the case that the $\mathrm{RG}$ effects are suppressed. Some conditions on the parameters to realize the QLC relation will be discussed. Finally, we propose a possible way to achieve the QLC relation by taking a non-symmetric form of the down Yukawa matrix.

The flavor mixings stem from the mismatch between the left-handed rotations of the up-type and down-type quarks, and the charged leptons and neutrinos. For our purpose, it is useful to work in a basis where the quark and lepton Yukawa matrices are related. In general, the quark Yukawa matrices $Y_{u}, Y_{d}$ are given by $Y_{u}=U_{u} Y_{u}^{\text {diag }} V_{u}^{\dagger}, \quad Y_{d}=U_{d} Y_{d}^{\text {diag }} V_{d}^{\dagger}$, from which the observable quark mixing matrix is deduced as $U_{\mathrm{CKM}}=U_{u}^{\dagger} U_{d}$. For the charged lepton sector, the Yukawa matrix is given by $Y_{l}=U_{l} Y_{l}^{d i a g} V_{l}^{\dagger}$. For the neutrino sector, we introduce one right-handed singlet neutrino per family which leads to the seesaw mechanism, according to which the light neutrino mass matrix after the breakdown of the electroweak symmetry is given by

$$
\begin{aligned}
M_{\nu} & =M_{\text {Dirac }} \frac{1}{M_{R}} M_{\text {Dirac }}^{T} \\
& =\left(U_{0} M_{\text {Dirac }}^{\text {diag }} V_{0}^{\dagger}\right) \frac{1}{M_{R}}\left(V_{0}^{*} M_{\text {Dirac }}^{\text {diag }} U_{0}^{T}\right),
\end{aligned}
$$

where $U_{0}$ and $V_{0}$ are the left-handed and right-handed mixing matrices of the Dirac neutrino mass matrix, respectively. We can then rewrite $M_{\nu}$ as follows

$$
M_{\nu}=U_{0} V_{M} M_{\nu}^{\operatorname{diag}} V_{M}^{T} U_{0}^{T},
$$

where $V_{M}$ represents the rotation of $M_{\text {Dirac }}^{\text {diag }} V_{0}^{\dagger} \frac{1}{M_{R}} V_{0}^{*} M_{\text {Dirac }}^{\text {diag }}$. The observable PMNS mixing matrix can then be written as [10]

$$
U_{\mathrm{PMNS}}=U_{l}^{\dagger} U_{\nu}=U_{l}^{\dagger} U_{0} V_{M}
$$

Note that equating the above expression for $U_{\text {PMNS }}$ with Eq. (2), we get the "CKM-like" form of $U(\lambda)$ :

$$
U^{\dagger}(\lambda)=U_{l}^{\dagger} U_{0} V_{M} U_{\text {bimax }}^{\dagger}
$$

In order for $U^{\dagger}(\lambda)$ to have "CKM-like" small mixing angles we have three possible choices of $U^{\dagger}(\lambda)$ :

$$
U^{\dagger}(\lambda)=\left\{\begin{array}{l}
U_{l}^{\dagger}, \\
U_{l}^{\dagger} U_{0}, \\
U_{l}^{\dagger} U_{0} V^{\prime},
\end{array}\right.
$$

where $V^{\prime}=V_{M} U_{\text {bimax }}^{\dagger}$. The first choice of $U^{\dagger}(\lambda)$ indicates that the maximal mixing angles in $U_{\text {bimax }}$ are cancelled out by the mixing angles in the combination of $U^{0} V_{M}$, while the second choice implies $V_{M}=U_{\text {bimax }}$. The last form is the most general one, which shows that the maximal mixing angles in $U_{\text {bimax }}$ are not completely cancelled out, and its actual form is not unique. In view of the quark-lepton unification, the second case is more natural than others because down-type (up-type) quarks are related with charged lepton (Dirac neutrino) 
sector in grand unification. In such a case, the form of $V_{M}$ is taken to be almost bi-maximal mixing because it is natural to suppose that the structure of the lepton mixing matrix to a leading order is the bi-maximal mixing, whereas the CKM matrix is an identity matrix, which corresponds to $U^{\dagger}(\lambda)=\mathrm{I}$ in Eq. (2), and then the QLC relation can emerge from quark-lepton unification. However, the bi-maximal mixing pattern of $V_{M}$ is not necessarily required. It is in fact possible to take $V_{M}$ to be small mixing or even identity matrix at GUT scale and then to generate two large mixing angles in the lepton mixing matrix by radiative magnification through evolving RG equations down to the weak scale [13, 14].

Now, let us consider how the PMNS mixing matrix given by Eq. (5) can be related with CKM mixing matrix in the context of quark-lepton unification.

\section{(A) Minimal quark-lepton unification}

Since the down-type quarks and the charged leptons are in general assigned into a multiplet in grand unification, we assume that the following simple relations hold in the minimal models of grand unification, $Y_{e}=Y_{d}^{T}, \quad Y_{u}=Y_{u}^{T}$. Then, we deduce that $U_{l}=V_{d}^{*}$ from which

$$
U_{\mathrm{PMNS}}=V_{d}^{T} U_{0} V_{M}
$$

From this expression for $U_{\mathrm{PMNS}}$, we see that the contribution of $U_{\mathrm{CKM}}$ may appear in $U_{\mathrm{PMNS}}$ if we further assume $Y_{\nu}=Y_{u}$ which can be realized in some larger unified gauge group such as $S O(10)$. Then, one can obtain [10]

$$
U_{\mathrm{PMNS}}=V_{d}^{T} U_{d} U_{\mathrm{CKM}}^{\dagger} V_{M} .
$$

In addition, requiring symmetric form of the down-type quark Yukawa matrix, we finally obtain

$$
U_{\mathrm{PMNS}}=U_{\mathrm{CKM}}^{\dagger} V_{M},
$$

where the mixing matrix $V_{M}$ has bi-maximal mixing pattern as explained above. In this way, $U_{\mathrm{PMNS}}$ can be connected with $U_{\mathrm{CKM}}$. We note that taking the bi-maximal mixing form of $V_{M}$ is equivalent to taking $M_{R}$ as follows, in the basis where $V_{0}$ is absorbed into the heavy Majorana neutrino field:

$$
M_{R}=\frac{1}{2}\left(\begin{array}{ccc}
\alpha m_{D_{1}}^{2} & \beta m_{D_{1}} m_{D_{2}} & -\beta m_{D_{1}} m_{D_{3}} \\
\beta m_{D_{1}} m_{D_{2}} & \left(\frac{1}{2} \alpha+\gamma\right) m_{D_{2}}^{2} & \left(-\frac{1}{2} \alpha+\gamma\right) m_{D_{2}} m_{D_{3}} \\
-\beta m_{D_{1}} m_{D_{3}} & \left(-\frac{1}{2} \alpha+\gamma\right) m_{D_{2}} m_{D_{3}} & \left(\frac{1}{2} \alpha+\gamma\right) m_{D_{3}}^{2}
\end{array}\right)
$$

where $\alpha=m_{1}^{-1}+m_{2}^{-1}, \beta=\left(-m_{1}^{-1}+m_{2}^{-1}\right) / \sqrt{2}, \gamma=m_{3}^{-1}$ and $m_{D_{i}}, m_{i}$ stand for the mass eigenvalues of Dirac mass matrix and light neutrino mass matrix, respectively.

To see whether the parametrization of $U_{\text {PMNS }}$ given by (10) can lead to the QLC relation (1), it is convenient to present $U_{\text {PMNS }}$ for the CP-conserving case as follows:

$$
\begin{aligned}
U_{\mathrm{PMNS}} & =U_{\mathrm{CKM}}^{\dagger} U_{23}^{m} U_{12}^{m} \\
& \equiv U_{23}\left(\theta_{23}\right) U_{13}\left(\theta_{13}\right) U_{12}\left(\frac{\pi}{4}-\theta_{12}\right),
\end{aligned}
$$

where $U_{12}^{m}$ and $U_{23}^{m}$ correspond to the maximal mixing between $(1,2)$ and $(2,3)$ generations, respectively. Then, the mixing angles $\theta_{i j}$ in the second line of Eq. (12) can be presented in 
terms of Wolfenstein parameter $\lambda$ as follows:

$$
\begin{aligned}
& \sin \theta_{12} \simeq \frac{1}{\sqrt{2}} \lambda+O\left(\lambda^{3}\right), \quad \sin \theta_{23} \simeq-\frac{1}{\sqrt{2}}\left(1-\frac{1}{2} \lambda^{2}\right), \\
& \sin \theta_{13} \simeq-\frac{1}{\sqrt{2}} \lambda .
\end{aligned}
$$

The solar neutrino mixing parameter $\sin \theta_{\text {sol }}$ in this parametrization becomes

$$
\sin \theta_{\text {sol }} \simeq \sin \left(\frac{\pi}{4}-\theta_{C}\right)+\frac{\lambda}{2}(\sqrt{2}-1) .
$$

Thus, we see that the neutrino mixing matrix (12) originating from the quark-lepton unification obviously leads to a shift of the relation (1). Numerically, the shift amounts to $\delta \theta_{\text {sol }} \simeq 3^{\circ}$ and thus $\delta \sin ^{2} \theta_{\text {sol }} \simeq 0.05$ which is more than $1 \sigma$ deviation from the recent measurement of the solar neutrino experiment. While a dedicated experiment to measure $\theta_{12}$ with a sensitivity of a few $\%$ to $\sin ^{2} \theta_{12}$ would be expected to confirm or rule out the deviation, we can expect that renormalization effects on the neutrino mixing matrix (12) may fill the gap between the QLC relation and the prediction (14)from high energy mixing matrix. Later, we will discuss the renormalization effect in detail.

(B) Realistic quark-lepton unification

Although the minimal quark-lepton unification can lead to an elegant relation between PMNS mixing and CKM mixing as shown in the above, it indicates undesirable mass relations between quarks and leptons at the GUT scale such as $m_{d}^{\text {diag }}=m_{l}^{\text {diag }}$. Recently, the following form for $U_{l}^{\dagger} U_{0}=U^{\dagger}(\lambda)$ has been suggested based on a well known empirical relation $\left|V_{u s}\right| \simeq \sqrt{\frac{m_{d}}{m_{s}}} \simeq 3 \sqrt{\frac{m_{e}}{m_{\mu}}}[18]$,

$$
U^{\dagger}(\lambda)=U_{l}^{\dagger} U_{0} \simeq\left(\begin{array}{ccc}
1 & -\frac{\lambda}{3} & \frac{1}{3} \theta \lambda^{2} \\
\frac{\lambda}{3} & 1 & 2 \theta \lambda \\
-\theta \lambda^{2} & -2 \theta \lambda & 1
\end{array}\right)
$$

where the deviation from unitarity is just of order $\theta \lambda^{3}$. This form of mixing matrix can be obtained by introducing the Higgs sector transforming under the representation 45 of $S U(5)$ or 126 of $S O(10)$ [19]. In this case, the solar neutrino mixing $\sin \theta_{\text {sol }}$ is then given by

$$
\sin \theta_{\text {sol }} \simeq \sin \left(\frac{\pi}{4}-\theta_{C}\right)+\frac{\lambda}{2}\left(\sqrt{2}-\frac{1}{3}\right) .
$$

Numerically, the deviation from the QLC relation amounts to $\delta \theta_{\text {sol }} \simeq 7^{\circ}$, much more than in the observed QLC relation. One possible way to generate the correct prediction for $\sin \theta_{\text {sol }}$ based on the lepton mixing with $U_{l}$ given by Eq. (15) is to abandon the exact bimaximal form of the neutrino mixing matrix $V_{M}$ and to consider the generic corrections to the bimaximal neutrino mixing matrix that can account for the QLC relation 18]. But, we consider an alternative possibility that the threshold corrections can diminish the deviation from the QLC relation.

Now, let us examine how the renormalization effects can diminish the deviation from the QLC relation. In general, the radiative corrections to the effective neutrino mass matrix can 
be presented as follows:

$$
\begin{aligned}
M_{\nu} & =I \cdot M_{\nu}^{0} \cdot I \\
& =I \cdot U_{\mathrm{CKM}}^{T} U_{\mathrm{bimax}}^{*} M_{D} U_{\mathrm{bimax}}^{\dagger} U_{\mathrm{CKM}} \cdot I \\
& =I \cdot U_{\mathrm{CKM}}^{T} U_{23}^{m^{*}} M_{D 12} U_{23}^{m \dagger} U_{\mathrm{CKM}} \cdot I
\end{aligned}
$$

where $M_{D}=\operatorname{Diag}\left[m_{1}, m_{2}, m_{3}\right], M_{D 12}=U_{12}^{m^{*}} M_{D} U_{12}^{m \dagger}$, and the matrix $I \equiv I_{A} \delta_{A B},(A, B=$ $e, \mu, \tau)$ stands for the radiative corrections. The correction $I$ generally consists of two parts $I=I^{R G}+I^{T H}$ where $I^{R G}$ are the renormalization group corrections which are explicitly presented in Ref. 20] and $I^{T H}$ are electroweak scale threshold corrections [15]. We note that the flavor blind interactions such as gauge interactions contribute to overall scale of neutrino masses whereas the charged lepton Yukawa interactions generate flavor dependent radiative corrections $I$ in the standard model (SM) and in MSSM. The typical size of RG corrections $I^{R G}$ is known to be about $10^{-6}$ in the SM and MSSM with small $\tan \beta$, and thus negligible. In addition, supersymmetry can induce flavor dependent threshold corrections related with slepton mass splitting which can dominate over the charged lepton Yukawa corrections [16]. Even if $I_{\tau}$ is the dominant contribution in SM, it is not guaranteed at all in MSSM due to the threshold corrections. We have numerically checked that RG evolution from the seesaw scale to the weak scale enhances the size of $\theta_{12}$ in the case that $\theta_{13}$ and $\theta_{23}$ are kept to be small and almost maximal mixing, respectively. Thus, the case of sizable RG effects is not suitable for our purpose. Instead, we examine whether the threshold corrections can be suitable for diminishing the deviation from the QLC relation while keeping $\theta_{23}$ nearly maximal and $\theta_{13}$ small in the case that $R G$ effect is negligible. To achieve our goal, we note that the contribution $I_{e}$ should be dominant over $I_{\mu, \tau}$ because only $I_{e}$ can lead to the right amount of the shift of $\theta_{12}$ while keeping the changes of $\theta_{23}$ and $\theta_{13}$ small. Taking $\left|I_{e}\right|>>\left|I_{\mu, \tau}\right|$, the neutrino mass matrix corrected by the leading contributions is rewritten as follows:

$$
M_{\nu} \simeq U_{\mathrm{CKM}}^{T} U_{23}^{m^{*}}\left[I_{D}+I_{e} \Lambda_{\lambda}^{*}\right] M_{D 12}\left[I_{D}+I_{e} \Lambda_{\lambda}^{\dagger}\right] U_{23}^{m \dagger} U_{\mathrm{CKM}}
$$

where $I_{D}$ is $3 \times 3$ identity matrix, and the matrix $\Lambda_{\lambda}$ is given up to $\lambda^{2}$ order by

$$
\begin{aligned}
\Lambda_{\lambda} & =\left(\begin{array}{ccc}
1 & -\frac{\lambda}{\sqrt{2}} & -\frac{\lambda}{\sqrt{2}} \\
-\frac{\lambda}{\sqrt{2}} & \frac{\lambda^{2}}{2} & \frac{\lambda^{2}}{2} \\
-\frac{\lambda}{\sqrt{2}} & \frac{\lambda^{2}}{2} & \frac{\lambda^{2}}{2}
\end{array}\right) \text { for minimal unification, } \\
& =\left(\begin{array}{ccc}
1 & -\frac{\lambda}{3 \sqrt{2}} & -\frac{\lambda}{3 \sqrt{2}} \\
-\frac{\lambda}{3 \sqrt{2}} & \frac{\lambda^{2}}{18} & \frac{\lambda^{2}}{18} \\
-\frac{\lambda}{3 \sqrt{2}} & \frac{\lambda^{2}}{18} & \frac{\lambda^{2}}{18}
\end{array}\right) \quad \text { for realistic unification. }
\end{aligned}
$$

Let us discuss how much the lepton mixing angles can be shifted by the renormalization effects. We first of all do numerical analysis in a model independent way based on the form given by Eqs. (19-20). For our numerical analysis, we take $\Delta m_{\text {sol }}^{2} \equiv m_{2}^{2}-m_{1}^{2} \simeq 7.1 \times 10^{-5} \mathrm{eV}^{2}$ and $\Delta m_{a t m}^{2} \equiv m_{3}^{2}-m_{2}^{2} \simeq 2.5 \times 10^{-3} \mathrm{eV}^{2}$. Regarding $M_{\nu}$ as the light neutrino mass matrix at low energy scale and varying the parameter $I_{e}$ and the smallest light neutrino mass $m_{1}$, we find which parameter set $\left(I_{e}, m_{1}\right)$ can lead to the QLC relation exactly and the results are presented in Table $\mathrm{I}$. We note that the required values for $I_{e}$ are negative. The first 


\begin{tabular}{|c|c|c|}
\hline & $(\mathrm{A})$ & $(\mathrm{B})$ \\
\hline$m_{1}(\mathrm{eV})$ & $I_{e}$ & $I_{e}$ \\
\hline \hline 0.15 & $-4.0 \times 10^{-5}$ & $-1.0 \times 10^{-4}$ \\
0.1 & $-8.5 \times 10^{-5}$ & $-2.2 \times 10^{-4}$ \\
0.05 & $-3.4 \times 10^{-4}$ & $-8.6 \times 10^{-4}$ \\
\hline
\end{tabular}

TABLE I: Parameter set $\left(I_{e}, m_{1}\right)$ leading to the QLC relation. The columns $(\mathrm{A})$ and $(\mathrm{B})$ correspond to the minimal unification and realistic case, respectively.

and the second column in Table I correspond to the minimal unification and realistic case, respectively. In our analysis, we have also checked that $\theta_{23}$ is almost unchanged, whereas the shift of $\theta_{13}$ is about $1^{\circ}$ for both cases (A) and (B). From Table I, we see that a larger value of $I_{e}$ is required to achieve the relation (1) as $m_{1}$ goes down.

How can we obtain such a value of $I_{e}$ while keeping $\left|I_{e}\right|>>\left|I_{\mu, \tau}\right|$ ? As shown in Ref. 17], it requires the existence of new states which gives a dominant contributions to $I_{e}$. In MSSM, it can easily be realized by taking into account the contribution of chargino (pure $\mathrm{W}$-ino). In the case of a diagonal slepton mass matrix in the same basis where the charged lepton mass matrix is diagonal, the contribution is presented by [17]

$$
I_{f} \simeq \frac{g^{2}}{32 \pi^{2}}\left(-\frac{1}{x_{f}}+\frac{1}{x_{\mu}}+\frac{x_{f}^{2}-1}{x_{f}^{2}} \ln \left(1-x_{f}\right)-\frac{x_{\mu}^{2}-1}{x_{\mu}^{2}} \ln \left(1-x_{\mu}\right)\right)
$$

where $x_{f}=1-\left(M_{f} / \tilde{m}\right)^{2}$ and $M_{f}, \tilde{m}$ are the $f$-th charged slepton mass and W-ino mass, respectively. We see that the size of $\left|I_{e}\right|$ is about 10 times larger than that of $\left|I_{\mu, \tau}\right|$ for $M_{e} \sim 2 M_{\mu, \tau}$, and the value of $I_{e}$ becomes negative and of the order of $10^{-4} \sim 10^{-3}$ for $x_{e} \gtrsim 0.65$, which are required to achieve the exact QLC relation at low energy. We also note that the contribution of the tau Yukawa coupling $Y_{\tau}$ in MSSM to $I_{\tau}$ can be as much as $I_{e}$ for large $\tan \beta$. Thus, our estimation is suitable for small $\tan \beta$ so that the contribution of $Y_{\tau}$ should be negligible compared to $I_{e}$.

In passing, we discuss a possible way to diminish the correction to QLC appeared in Eq. (14). While we have considered the case with symmetric form of fermion Yukawa matrices so far, non-symmetric types of $Y_{d}$ and $Y_{l}$ are generally allowed. Then, the term $V_{d}^{T} U_{d}$ in Eq. (9) is not trivial unity matrix in the non-symmetric Yukawa structure and it can generate additional correction to the solar neutrino mixing parameter $\sin \theta_{\text {sol }}$, which can diminish the correction to QLC. We have checked that $\sin \theta_{\text {sol }} \simeq \sin \left(\pi / 4-\theta_{C}\right)$ for the case of the minimal unification (A) when $V_{d}^{T} U_{d}$ has the form in the leading order of $\lambda$,

$$
V_{d}^{T} U_{d}=\left(\begin{array}{ccc}
1 & (1-\sqrt{2}) \lambda & -(1-\sqrt{2}) \lambda \\
-(1-\sqrt{2}) \lambda & 1 & 0 \\
(1-\sqrt{2}) \lambda & 0 & 1
\end{array}\right)
$$

For the realistic case (B), one can obtain the QLC relation by taking the matrix form (22) but replacing $\lambda$ with $\lambda / 3$. We note that in order to achieve the QLC relation at low energy scale in those cases, renormalization effects should be negligibly small. But, it is quite arbitrary to generate the form of $V_{d}^{T} U_{d}$ given above from some underlying symmetries or flavor structure. 
In summary, while the QLC relation, $\theta_{\text {sol }}+\theta_{C}=\frac{\pi}{4}$, itself can be an evidence for the quark-lepton unification, it can be a coincidence in the sense that the relation achieved at low energy in the framework of grand unification strongly depends on the renormalization effects whose size can vary with the choice of parameter space. In this paper, we have found that the lepton mixing matrix derived from quark-lepton unification can lead to a shift of the complementarity relation at a high energy. While the renormalization group effects generally lead to additive contribution on top of the shift, we show that the threshold corrections which may exist in the supersymmetric standard model diminish it, so we can achieve the complementarity relation at a low energy. In addition, we commented on how to achieve the QLC relation by taking a non-symmetric form of the down Yukawa matrix.

We would like to thank G. Cvetic for careful reading of the manuscript and his valuable comments. The work of S.K.K was supported in part by BK21 program of the Ministry of Education in Korea and in part by KOSEF grant R02-2003-000-10085-0. The work of C.S.K. was supported in part by CHEP-SRC Program and in part by Grant No. R02-2003-00010050-0 from BRP of the KOSEF. The work of J.L. was supported in part by BK21 program of the Ministry of Education in Korea and in part by Grant No. F01-2004-000-10292-0 of KOSEF-NSFC International Collaborative Research Grant.

[1] S. Hukuda et al. [Super-Kamiokande Collab.], Phys. Rev. Lett. 86, 5656 (2001); Phys. Lett. B 539, 179 (2002).

[2] Q. Ahmad et al. [SNO Collab.], Phys. Rev. Lett. 87, 071301 (2001); Phys. Rev. Lett. 89, 011301 (2002); S. Ahmed et al. [SNO Collab.], arXiv:nucl-ex/0309004.

[3] G. L. Fogli et al., hep-ph/0310012; A. Bandyopadhyay et al., hep-ph/0309174.

[4] M. Raidal, hep-ph/0404046.

[5] H. Minakata and A. Yu. Smirnov, hep-ph/0405088

[6] P. H. Frampton and R. N. Mohapatra, hp-ph/0407139

[7] C. Giunti and M. Tanimoto, Phys. Rev. D 66, 053013 (2002).

[8] W. Rodejohann, Phys. Rev. D 69, 033005 (2004).

[9] P. H. Frampton, S. T. Petcov and W. Rodejohann, Nucl. Phys. B 68731 (2004).

[10] A. Datta, F-S Ling and P. Ramond, Nucl. Phys. B671, 383 (2003); P. Ramond, hep-ph/0405176.

[11] F. Vissani, hep-ph/0978483 V. D. Barger, S. Pakvasa, T. J. Weiler and K. Whisnant, Phys. Lett. B 437, 107 (1998); A. J. Baltz, A. S. Goldhaber and M. Goldhaber, Phys. Rev. Lett. 81, 5730 (1998); R. N. Mohapatra and S. Nussinov, Phys. Rev. D 60, 013002 (1999); Phys. Lett. B 441, 299 (1998); S. K. Kang and C. S. Kim, Phys. Rev. D 59, 091302 (1999); H. Fritzsch and Z.-z. Xing, Prog. Part. Nucl. Phys. 45, 1 (2000) and references therein.

[12] J. A. Casas, J. R. Espinosa, A. Ibarra and I. Navarro, Nucl. Phys.573, 652 (2000); N. Haba, Y. Matsui and N. Okamura, Eur. Phys. C 17, 513 (2000); S. Antusch, J. Kersten, M. Lindner and M. Ratz, Nucl. Phys. 674, 401 (2003); S. Antusch, J. Kersten, M. Lindner, M. Ratz and M. A. Schmidt, hep-ph/0501272,

[13] K.R.S.Balaji, A.S. Dighe, R. N. Mohapatra, M. K. Parida, Phys. Lett. B 481, 33 (2000); Phys. Rev. Lett. 84, 5034 (2000); K.R.S.Balaji, R. N. Mohapatra, M. K. Parida and E. A. Pascos, Phys. Rev D 63, 113002 (2001); R. N. Mohapatra, M. K. Parida and G. Rajasekaran, 
Phys. Rev. D 69, 053007 (2004).

[14] S. Antusch and M. Ratz, JHEP 0211, 010 (2002); C. Hagedorn, J. Kersten and M. Lindner, Phys. Lett. 597, 63 (2004).

[15] P. H. Chankowski and S. Pokorski, Int. J. Mod. Phys. A 17, 575 (2002); P. H. Chankowski and P. Wasowicz, Eur. Phys. J. C 23, 249 (2002).

[16] E. J. Chun and S. Pokorsky, Phys. Rev. D 62, 053001 (2000); B. Brahmachari and E. J. Chun, Phys. Lett. B 596, 184 (2004).

[17] P. H. Chankowski, A. N. Ioannisian, S. Pokorski and J. W. F. Valle, Phys. Rev. Lett. 86, 3488 (2001).

[18] J. Ferrandis and S. Pakvasa, hep-ph/0409204 hep-ph/0412038.

[19] H. Georgi and . Jarlskog, Phys. Lett. B 86, 297 (1979); K. S. Babu and S. M. Barr, Phys. Rev. Lett. 85, 1170 (2000).

[20] J. Ellis and S. Lola, Phys. Lett. B 458, 310 (1999). 